\title{
Valoración del registro de la información del plan de cuidados y de la trayectoria clínica de la implantación del catéter peritoneal
}

\author{
Puiguert Vilalta Carme* \\ Muriana Zamudio Carme** \\ García Garrido Lluïsa*** \\ Navarro Ferrer Encarnación ****
}

\author{
Hospital Universitario Dr. Josep Trueta. Girona \\ * Supervisora de la unidad de diálisis \\ **Enfermera de la unidad de diálisis \\ ***Profesora adjunta de la Escuela de Enfermería \\ de la Universidad de Girona \\ ****Enfermera de la unidad de cirugía
}

\begin{abstract}
RESUMEN
El objetivo de este estudio es elaborar y valorar la implantación del plan de cuidados y la trayectoria clínica de la colocación del catéter peritoneal para corroborar que la trayectoria clínica es un buen registro para enfermería.
\end{abstract}

Para elaborar el plan de cuidados hemos realizado la valoración según las necesidades de V. Henderson. Para la formulación de los diagnósticos hemos utilizado la Taxonomia II de la NANDA y para las intervenciones y los resultados hemos relacionado el NIC y el NOC. La trayectoria clínica resalta el proceso por el cual pasa el enfermo desde la implantación del catéter (2 días de hospitalización), el control ambulatorio en la unidad de diálisis peritoneal cada 24 horas (8 días), hasta el inicio definitivo del tratamiento. Se ha diseñado una hoja para registrar las incidencias de la trayectoria clínica que nos permita detectar las desviaciones para poder utilizar las medidas correctoras. La trayectoria clínica cumple las mismas características que los registros.

Estos instrumentos de gestión han facilitado la calidad de los cuidados, teniendo en cuenta acciones centradas en los resultados y permiten conocer la estimación de los costes generados por la enfermería.

\author{
Correspondencia: \\ Carme Puiguert Vilalta \\ Unidad de diálisis \\ Hospital Universitario Dr. Josep Trueta de Girona \\ Avenida de Francia s/n \\ 17007 Girona
}

\author{
PALABRAS CLAVE: VALORACIÓN \\ DIÁLISIS PERITONEAL \\ PLAN DE CUIDADOS \\ TRAYECTORIA CLÍNICA
}

\section{EVALUATION OF THE RECORDING OF INFORMA- TION ON THE CARE PLAN AND CLINICAL PATH IN THE IMPLEMENTATION OF A PERITONEAL CATHETER}

\section{SUMMARY}

The aim of this study is to draw up and evaluate the implementation of the care plan and the clinical path in the placing of a peritoneal catheter to check that the clinical path is a good record for nursing.

To draw up the care plan, we have conducted the evaluation on the basis of the needs of V. Henderson. For diagnoses we have used NANDA Taxonomy II and for interventions and outcomes we have related NIC and NOC. The clinical path highlights the process undergone by the patient from implementation of the catheter ( 2 days in hospital), control in the peritoneal dialysis unit every 24 hours ( 8 days) until treatment definitively commences. A sheet has been designed to record incidences in the clinical path that lets us detect any deviations in order to apply corrective measures. The clinical path has the same characteristics as the records. 
These management instruments have facilitated the quality of care, taking into account outcome-focused actions and make it possible to estimate the costs generated by nursing.

\section{KEY WORDS: EVALUATION PERITONEAL DIALYSIS \\ CARE PLAN CLINICAL PATH}

\section{INTRODUCCIÓN}

La recogida y registro de la información es el punto de partida en la elaboración del plan de cuidados, que es un protocolo de enfermería en el que se definen los problemas, las técnicas, los cuidados y los tratamientos más frecuentes que pueden encontrarse los enfermos en una situación específica, y también recoge la educación sanitaria. En su realización, no sólo se describen los problemas más usuales si no que también engloban las intervenciones con sus actividades más prioritarias, donde los resultados de los mismas nos conducen a poder afirmar la calidad de los cuidados ${ }^{(1)}$, convirtiéndose no únicamente en uno de los retos más importantes para la enfermera, si no también para el equipo clínico que participa en la elaboración, la confección, la realización y la puesta en marcha del plan de cuidados. A la vez nos puede permitir medir, controlar y estimar los costes generados por la enfermería durante el proceso.

Actualmente los planes de cuidados tienen como objetivo mejorar la práctica asistencial y evitar las diferencias de criterios de los profesionales en la atención al paciente. En la realidad un plan de cuidados nos ayuda especialmente a unificar criterios entre los distintos profesionales que participan en el proceso y a la vez éste nos sirve de guía(2).

Se garantiza así un trato homogéneo al paciente sea cual sea el profesional que le atienda, un punto muy importante en el trabajo de enfermería, ya que la naturaleza de nuestro trabajo exige una jornada a turnos para la continuidad de los cuidados. El hábito de la recogida y estructuración de la información por parte de la enfermería se materializa en el empleo de registros y protocolos enfermeros. Éstos deben estar diseñados correctamente para asegurar que responden a la necesidad para la cual han sido creados, ser flexibles y modificables en caso de necesitar variar algún aspecto de los mismos una vez instaurados, por lo que su evaluación ha de ser continua una vez establecidos y realizados por los mismos profesionales que hacen uso diario de ellos ${ }^{(3)}$.

El 30\% de los pacientes diagnosticados de insuficiencia renal crónica terminal (IRCT) que inician programa de tratamiento

substitutivo de la insuficiencia renal (TIRS), escogen la diálisis peritoneal (DP)(4).

El paciente que realiza DP debe de ser portador de un catéter en la cavidad peritoneal. La técnica de colocación de dicho catéter la realizan los cirujanos mediante laparoscopia, que consideran esta intervención como cirugía mayor ambulatoria y también, en general, piensan que la movilidad no es un factor condicionante del buen funcionamiento del catéter. En cambio los profesionales de nefrología pensamos que el paciente debe mantener un reposo relativo dependiendo de su constitución. Tras un amplio debate entre cirujanos y nefrólogos se llegó a un acuerdo, estos pacientes ingresaran en la unidad de cirugía general del hospital, el día anterior a la colocación del catéter peritoneal y se les dará el alta 24 horas después de la implantación del catéter debiendo guardar un reposo relativo. En caso de que no haya camas en cirugía general ingresarán en la unidad de nefrología, por lo que tenemos la necesidad de protocolizar la atención de los pacientes en esta circunstancia, para conseguir el consenso sobre la idoneidad de las actuaciones.

El objetivo de este estudio es elaborar y valorar la implantación del plan de cuidados y la trayectoria clínica de la colocación del catéter peritoneal, valorando la utilidad de la trayectoria clínica como registro de enfermería.

\section{METODOLOGÍA}

Para la elaboración del plan de cuidados de la colocación del catéter peritoneal se realizó un estudio descriptivo y retrospectivo. Se creó un grupo de trabajo formado por la supervisora, la enfermera de la unidad de diálisis que llevan a cabo la DP y una enfermera experta en metodología. El período del estudio fue de febrero de 2003 a noviembre de 2004 y comprendió desde la elaboración (febrero de 2003 a octubre de 2003) del plan de cuidados y de la trayectoria clínica hasta la implantación (noviembre de 2003 a noviembre de 2004) de la trayectoria en las unidades de hospitalización y diálisis. Se incluyeron en el estudio a todos los pacientes que iniciaron el programa de educación sanitaria (PES) y que escogieron como TIRS la DP, en el Hospital Universitario Dr .Josep Trueta de Girona.

El plan de cuidados consta de dos partes diferenciadas entre sí. En la primera se identifican los problemas interdependientes, en ellos se engloba los problemas biomédicos que la enfermera tiene que conocer para vigilar, controlar e identificar todas las complicaciones potenciales. En la segunda parte se describen los diagnósticos de enfermería. La enfermera se centra en la valoración de las 14 necesidades básicas de la persona (las necesidades alteradas fueron eliminación, dormir y descansar, higiene, estado de la piel y 
tegumentos, comunicación y aprender), las manifestaciones de dependencia y el posterior análisis de los datos. Para el anunciado del problema se utilizó la taxonomía diagnóstica II de la NANDA ${ }^{(5,6,7)}$. También se han descrito las diferentes intervenciones enfermeras que pretenden conseguir una acción terapéutica mediante una serie de actividades que se deben llevar a término en una intervención para conseguir un resultado concreto.

Para la clasificación de las intervenciones de enfermería se ha utilizado el $\mathrm{NIC}^{(8)}$ y para los resultados esperados con indicadores cuantitativos para medir la consecución de los mismos utilizamos la clasificación $\mathrm{NOC}^{(9)}$. Pretendiendo describir en este plan de cuidados unas actividades realistas y fácilmente asumibles, intrínsecamente relacionadas con la estructura física, los recursos humanos y materiales del lugar de trabajo.

El grupo de trabajo para elaborar la trayectoria clínica, fue asesorado por el nefrólogo responsable de la unidad de diálisis peritoneal, un cirujano y una enfermera de cirugía. La implantación de los catéteres peritoneales fue realizada por los cirujanos, mediante la técnica laparoscópica y el Y-Tec.

Esta trayectoria clínica consiste en la estandarización de manera secuencial de todas las actividades, proporcionando un orden y un método a la atención, consiguiendo una optimización de la efectividad y eficiencia. Eengloba la definición del paciente tipo, describe las necesidades de salud alteradas, así como los diagnósticos de enfermería que se derivan. También incluye un mapa con todas las actividades que hay que llevar a término durante el proceso que el enfermo pasa desde la implantación del catéter (2 días de hospitalización), el control ambulatorio en la unidad de DP cada 24 horas (8 dias), hasta el inicio definitivo del tratamiento.

Se elaboró una hoja para registrar las variaciones respecto al estándar para así utilizar las medidas correctoras y por último se incluyeron las recomendaciones al alta del paciente.

Se comunicaba a la enfermera o a la supervisora de diálisis el ingreso del paciente, éste ingresaba en una de las dos unidades de cirugía general o en la unidad de nefrología y se entregaba la trayectoria clínica a la enfermera responsable del ingreso del paciente. Previamente la supervisora de diálisis había presentado a las supervisoras de las unidades de cirugía general y de nefrología el plan de cuidados y explicó como debían recoger los datos de la trayectoria clínica (los 2 días de hospitalización). Una vez dado el alta al paciente, la historia se depositaba en la unidad de diálisis peritoneal para continuar el aprendizaje del paciente.

\section{RESULTADOS}

Se cumplimentaron 9 trayectorias clínicas, 5 pacientes ingresaron en la unidad de nefrología y 4 en cirugía, 2 en cada una de las unidades de cirugía general. 9 fueron varones y 1 hembra, con una edad media de 42 años y un rango comprendido entre 27 y 55 años. Los catéteres implantados fueron todos de Tenckoff de Swan.Neck con 2 manguitos de dacron.

\section{Valoración de la cumplimentación de la trayectoria clí- nica:}

- 2 días de hospitalización: Todos los pacientes tenían valorados los bloques que correspondían al pre y al post operatorio. Uno de ellos no tenía cumplimentada ninguna actividad que correspondiera a los dos días de ingreso en la planta de nefrología. Dos pacientes respetaron el reposo absoluto durante los dos días de ingreso post implantación del catéter. En 4 pacientes el reposo fue absoluto el primer día y relativo en el segundo día. En tres el reposo fue relativo; la necesidad de tomar ansiolíticos fue valorada a 4 pacientes, para los demás pacientes fue suficiente con las medidas de comodidad y ajuste del medio ambiente (luz, ruido, horario). Las actividades que corresponden al diagnóstico de déficit de autocuidado fueron aplicadas a 7 pacientes. Siete pacientes fueron instruidos para evitar el estreñimiento, de ellos 4 necesitaron la administración de laxantes.

- 8 días de control ambulatorio cada 24 h. en DP. Al tercer día 1 paciente aun continuaba en reposo absoluto por dolor en hombro derecho y molestias abdominales, otro paciente continuaba en reposo relativo. De los pacientes que tomaron laxantes los días de hospitalización dos necesitaron seguir tratándose durante una semana complementando con dieta de fibra (controlando el $\mathrm{K}$ en el plasma). Dos pacientes necesitaron tomar ansiolíticos durante unas semanas. No tuvieron ningún problema para tocar el catéter y el orificio ocho pacientes, mientras que un paciente estaba tan angustiado que solamente fue capaz de mirar. Todos los pacientes describieron bien la dieta a seguir a sí como el tratamiento medicamentoso.

La hoja de variaciones reflejó que un paciente retrasó una semana su PES nivel III debido a una gran angustia y miedo. Por el contrario otro paciente al iniciar el PES nivel III ya conocía muchos conceptos que se le habían explicado en la información del tratamiento sustitutivo. Respecto de las recomendaciones al alta se refleja la actividad, dentro de sus posibilidades, que beneficiaron a la eliminación (deposición) y al sueño y descanso. Realizar cura diaria o cada dos días del orificio del catéter, así como una buena fijación del mismo. 
La trayectoria clínica cumple con las características básicas que deben seguir los registros para asegurar su utilidad, ya que:

- Garantiza el seguimiento de los cuidados

- Identifica al paciente y al profesional

- Ubica en espacio y tiempo

- Emplea una terminología unificada

- Permite una utilización posterior

\section{DISCUSIÓN Y CONCLUSIONES}

Una de las complicaciones que nos hemos encontrado en la elaboración del plan de cuidados y la trayectoria clínica es la utilización de los diferentes términos traducidos que muchas veces no se complementan con nuestra cultura del cuidar y esto supone la dificultad en la utilización, la descripción y la expresión del lenguaje escrito de estas metodologías con nuestra práctica clínica. No obstante, la mayor dificultad fue al relacionar los tres conceptos como son NANDA, NIC y NOC debido a que todavía somos debutantes en esta metodología de relación. Otra reflexión que nos gustaría plasmar en este trabajo es la necesidad de utilizar de un lenguaje común y único para las enfermeras, donde se use una terminología estandarizada en relación con los diagnósticos enfermeros, intervenciones y resultados.

Los planes de cuidados estandarizados, las trayectorias clínicas y otros instrumentos de gestión están siendo instaurados actualmente en muchos centros sanitarios como herramienta de trabajo diario del profesional de enfermería.

Las enfermeras que hemos utilizado la trayectoria clínica como herramienta de trabajo diario en hospitalización y diálisis pensamos que concuerda el proceso con la realidad y que se adapta al medio donde se utiliza. También creemos que la trayectoria clínica es fácil y rápida de cumplimentar como registro de enfermería y que cumple con las características básicas de los registros.
Estos instrumentos de gestión suponen asegurar la calidad de los cuidados teniendo en cuenta acciones centradas en los resultados y también nos da a conocer la estimación de los costes generados por la enfermería.

\section{AGRADECIMIENTO}

Al Dr. Pere Torguet y al Dr. Josep Roig.

\section{BIBLIOGRAFIA}

1. Tomás Vidal AM. Metodología para la elaboración de planes de cuidados. Enfermería Clínica 1994; 4 (1): 36-38.

2. Tomás Vidal AM. Conceptos básicos para la elaboración de planes de cuidados. Enfermería Clínica 1994; 4 (1): 2835.

3. Lavado Núñez ME, Márquez Arangudez MP, Dama Sosa C, y Manfredi López MJ. Registros y Protocolos. Rev. Gestión de Cuidados. HYGIA 2004; 57:10-14.

4. Puigvert Vilalta C, Muriana Zamudio C, Garcia Garrrido MLl. Valoración del Programa de Educación Sanitaria de los pacientes con IRC Rev Soc. Esp Enfer Nefrol 2002; (5) 4:14-17.

5. NANDA. Diagnósticos Enfermeros: Definiciones y Clasificaciones 2001-2002. Ed. Harcourt: Madrid. 2001.

6. Ugalde Apalategi M, Rigol i Cuadra A. Diagnóstico de Enfermería. Taxonomía Nanda Ed. Masson; 1997.

7. Luis Ma T. Diagnósticos Enfermeros. Un instrumento para la práctica asistencial. 3 Ed. Harcourt-Brace; 1997.

8. McCloskey J,C., Burlechek G.M. Proyecto de Intervenciones IOWA. NIC. Clasificación de Intervenciones de Enfermería (CIE). 3 ed. Ed. Harcourt-Mosby; 2001.

9. Johnson M, Maas M, Moorhead S. Proyecto de resultados IOWA. NOC. Clasificación de Resultados de Enfermería (CRE). 2ํㅡ. Ed. Harcourt-Mosby; 2001. 

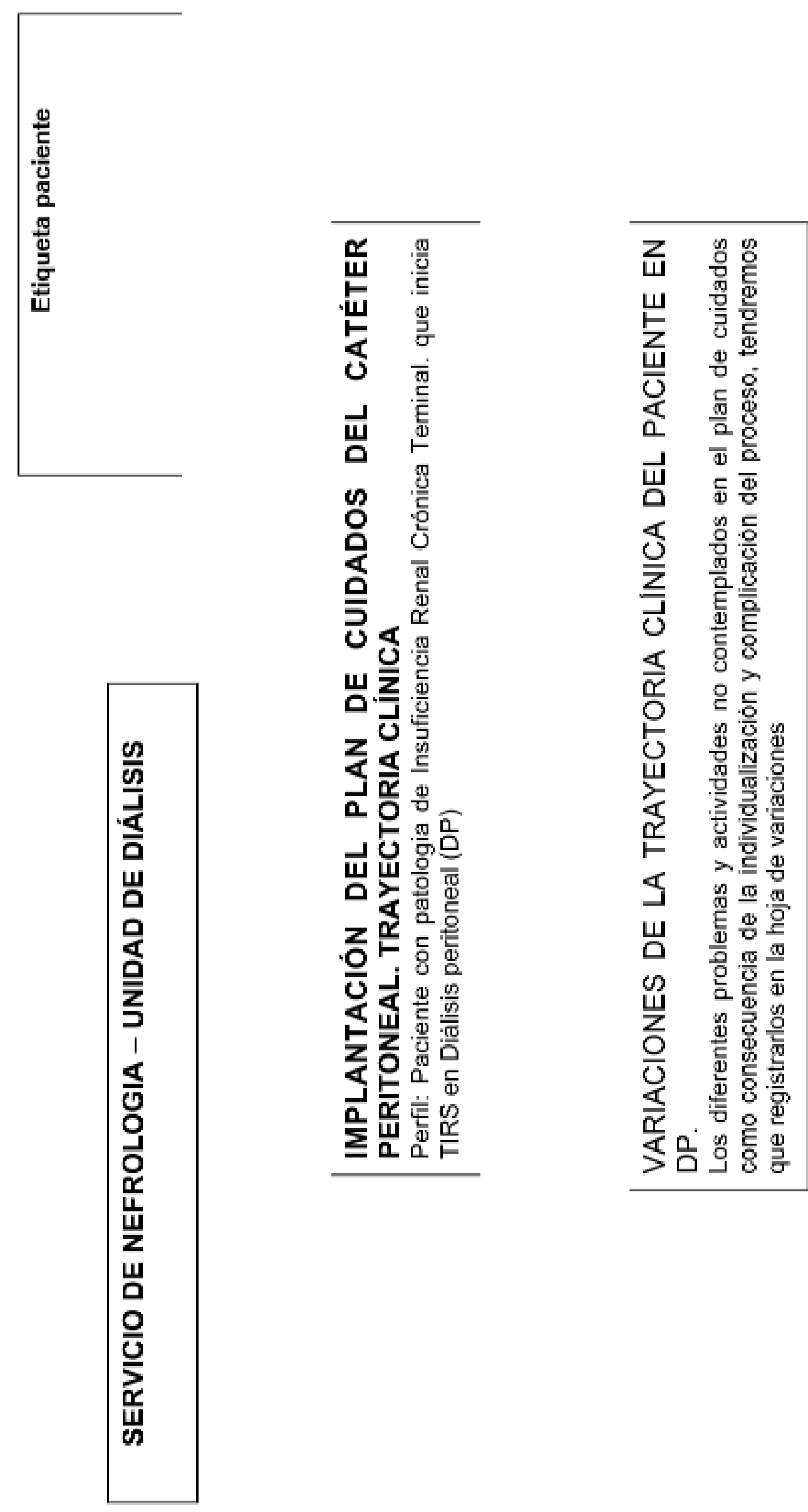


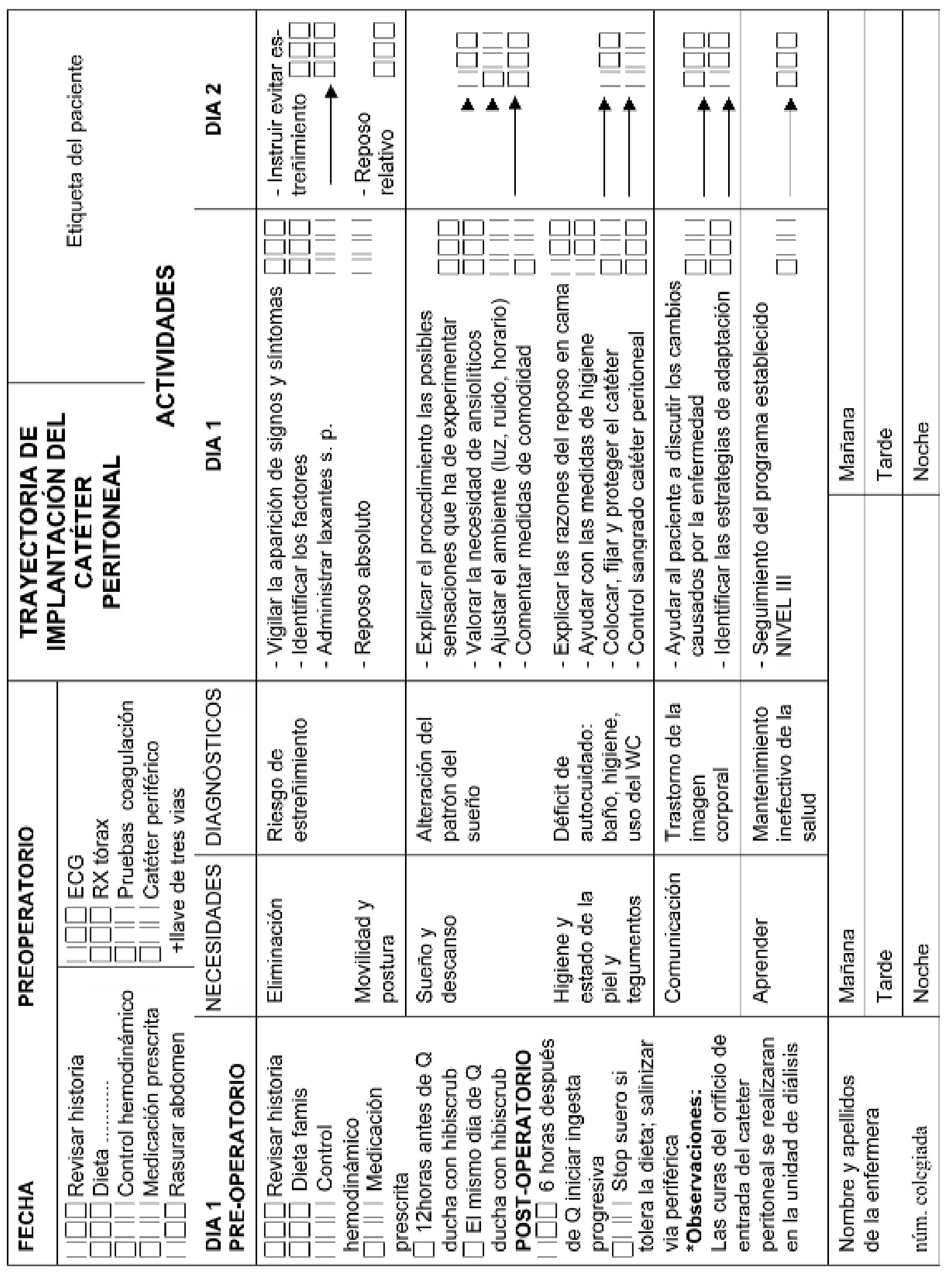




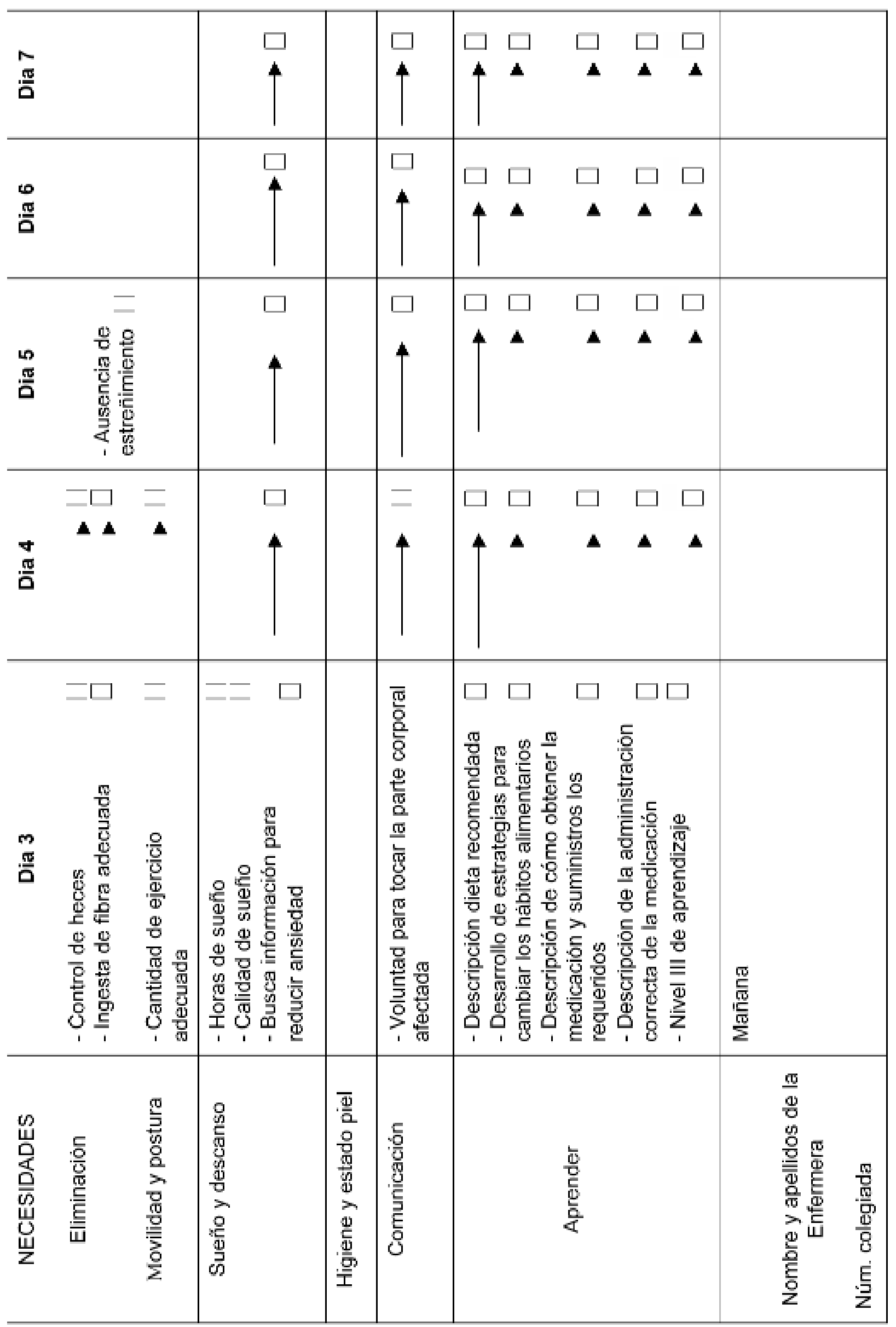




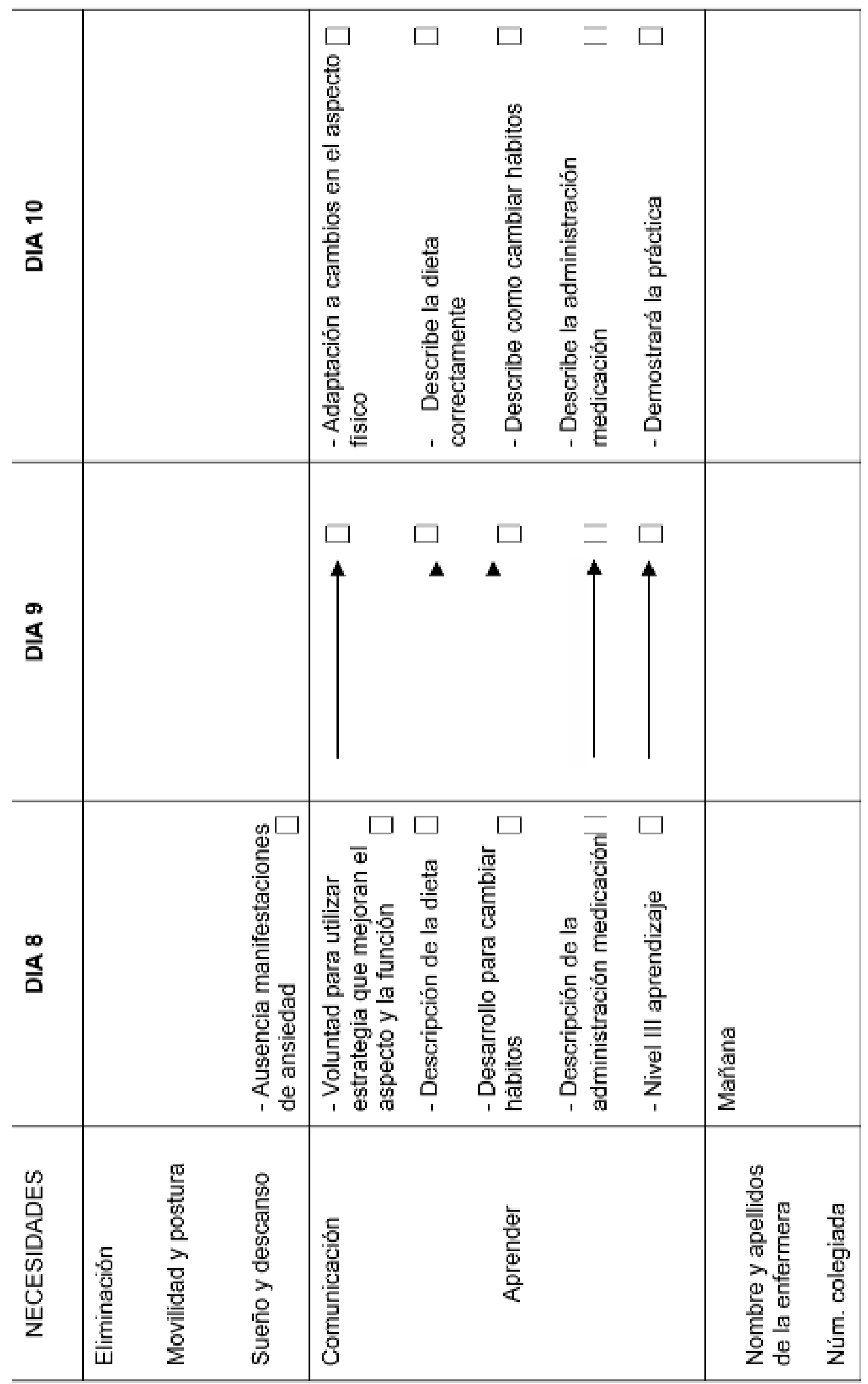




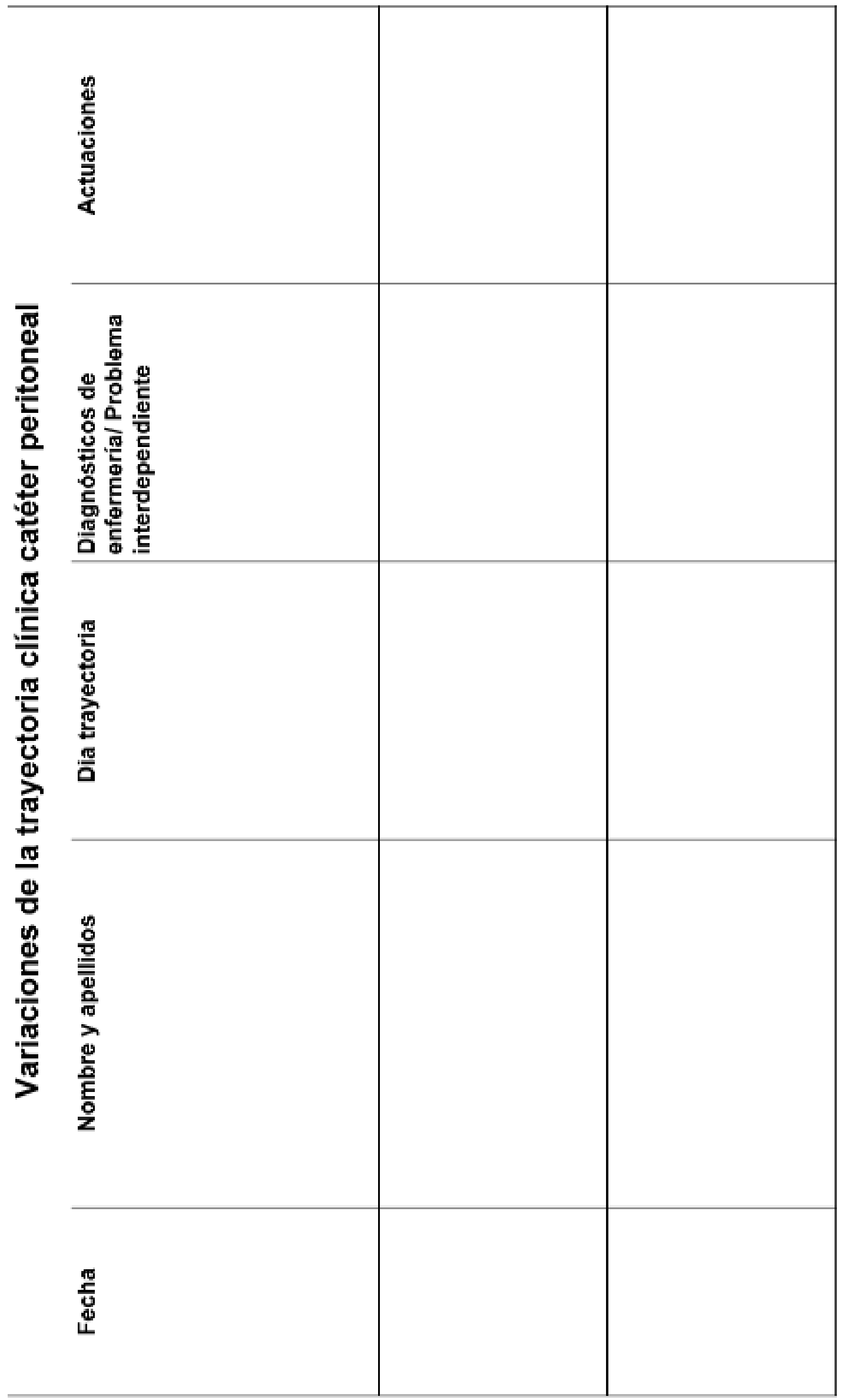

